\title{
QoS-TI: Um Framework Adaptável para Qualidade do Serviço de Suporte de TI nos Institutos Federais
}

\author{
Cristiano D. da Silva ${ }^{1,2}$, Alexandre Marcos de Lins $\operatorname{Vasconcelos}^{1}$ \\ ${ }^{1}$ Centro de Informática - Universidade Federal de Pernambuco (UFPE) \\ Caixa Postal 7.851 - 91.501-970 - Recife - PE - Brazil \\ ${ }^{2}$ Instituto Federal de Educação, Ciência e Tecnologia de Goiás (IFG) \\ Av. Assis Chateaubriand, no 1.658 - 74.130-102 - Goiânia - GO - Brazil \\ $\{$ cds 3, amlv\}@cin.ufpe.br
}

\begin{abstract}
This article presents QoS-TI, a framework that aims to support the implementation or improvement of Service Desks in Federal Institute (IFs). Structured through a life cycle based on the IDEAL model and a process Toolbox, the QoS-TI framework incorporates several practices identified in the quality and service models ITIL, ISO 9001, ISO 20000, CMMI Services and MR-MPS-SV, and provides a "how-to" approach, rather than a "what to do" approach. After its development, the framework was evaluated by IFs's specialists. The aspects evaluated received positive opinions from most specialists.
\end{abstract}

Resumo. Este artigo apresenta o QoS-TI, um framework que tem por objetivo apoiar a implantação ou a melhoria de Centrais de Serviços de TI nos Institutos Federais (IFs). Estruturado através de um ciclo de vida baseado no modelo IDEAL e de um Toolbox de processos, o framework QoS-TI incorpora diversas práticas identificadas nos modelos de qualidade e serviços ITIL, ISO 9001, ISO 20000, CMMI Services e MR-MPS-SV, e fornece uma abordagem focada em "como fazer", ao invés de "o quê fazer". Após o seu desenvolvimento, o framework foi avaliado por especialistas dos IFs. Os aspectos avaliados receberam opiniões positivas da maioria dos especialistas.

\section{Introdução}

Os Institutos Federais de Educação, Ciência e Tecnologia (IFs) expandiram-se por meio da estrutura multicampi, o que acarretou profundas mudanças em suas organizações administrativa e acadêmica (Brasil, 2015). Em consequência dessa ampliação ocorreu o aumento de demandas por serviços, sistemas e soluções de TI; o que ocasionou diversos problemas. Ficou evidente a necessidade de desenvolver uma proposta que apoie os IFs a melhorar o serviço de suporte de TI. A Central de Serviços de TI é uma alternativa, pois implementa a interface única entre usuários e o setor de TI, todavia com papel mais amplo do que apenas o de suporte técnico, pois engloba processos, pessoas e tecnologias voltadas para o gerenciamento de serviços de TI.

Este trabalho teve como objetivo desenvolver um framework, com abordagem prática em "como fazer", que oriente a implantação e/ou a melhoria de Centrais de Serviços de TI dos Institutos Federais. A proposta buscou incorporar as boas práticas 
identificadas nos modelos de qualidade e serviços pesquisados, criando uma dinâmica de implantação e melhoria de Centrais de Serviços de TI através de uma estrutura composta por um Ciclo de Vida, baseado no modelo IDEAL (Mcfeeley, 1996), e de um Toolbox de processos, estruturado conforme as sete dimensões do Extended Process Maturity Framework - EPMF (Rudd, 2010).

A justificativa desta pesquisa é decorrente da carência de orientações de implantação e/ou melhoria de uma Central de Serviços de TI sob o ponto de vista prático, pois as principais normas, modelos e trabalhos estudados concentram "no que tem que ser feito", pois apresentam os requisitos que devem ser atendidos para a entrega do serviço com qualidade, mas pouco abordam o "como", isto é, não mostram o caminho para a implantação da Central de Serviços. A avaliação da proposta foi realizada por especialistas dos IFs, e com isso, buscou-se mostrar que o framework é adequado para implantar e/ou melhorar uma Central de Serviços.

Além desta seção introdutória, o artigo está organizado da seguinte forma: a seção 2 apresenta a revisão da literatura sobre qualidade de serviços, normas e modelos de gerenciamento de serviços; a seção 3 apresenta trabalhos relacionados; a seção 4 apresenta o framework QoS-TI; a seção 5 apresenta a avaliação do framework, realizada por especialistas; por fim, a seção 6 apresenta as considerações finais.

\section{Fundamentação Teórica}

Inúmeras são as definições dadas ao termo "qualidade". Feigenbaum (1961) enfatiza que qualidade não é apenas uma técnica para eliminar defeitos nas operações, é um sistema de gestão e um compromisso com a excelência. Juran (1992) define qualidade como adequação ao uso, ou seja, são características que atendem as necessidades do cliente e promovem a sua satisfação. Ishikawa (1993) afirma que a qualidade se fundamenta no cliente, com a entrega de produtos/serviços que atendam às suas necessidades. Para a Softex (2015), qualidade é um fator crítico de sucesso para o setor de serviços, já o CMMI Institute (2010) afirma que a qualidade de um sistema ou produto é altamente influenciada pela qualidade do processo utilizado para desenvolvêlo e mantê-lo. No que se refere ao termo "serviço" podemos citar a definição da Information Technology Infrastructure Library - ITIL (OGC, 2007), "serviço é um meio de entregar valor aos clientes, facilitando os resultados que eles querem alcançar sem que tenham que assumir custos e riscos específicos". Nesse sentido, o conceito de qualidade de serviço utilizado neste trabalho está relacionado à qualidade do processo de gerenciamento do serviço e ao atendimento das necessidades dos clientes.

Várias normas e modelos para melhoria da qualidade de serviços foram criadas e aperfeiçoadas ao longo dos anos, e entre eles podemos destacar: a Information Technology Infrastructure Library (OGC, 2007), a ISO 9001 (ABNT, 2015), a ISO/IEC 20000 (ABNT, 2011), o modelo de referência MR-MPS-SV (SOFTEX, 2015) e o Capability Model Integration for Services (CMMI-SVC, 2010).

A ITIL é uma compilação das melhores práticas utilizadas para o gerenciamento de serviços de tecnologia de informação, obtidas em consenso após décadas de observação prática, pesquisa e trabalho de profissionais de TI em todo mundo (Fernandes e Abreu, 2014). A biblioteca descreve sobre particularidades da função Central de Serviços, dentre as quais podemos destacar: escolha e treinamento de 
pessoal, definição de responsabilidades, tipos de estruturas, ponto único de contato, medição de desempenho e pesquisa de satisfação. A norma ISO 9001 tem foco nas especificações dos requisitos do sistema de gestão da qualidade da organização. A norma ISO/IEC 20000 tem foco na padronização, no cliente, nos processos e na melhoria contínua dos processos. O CMMI-SVC (Capability Maturity Model Integration for Services) é um conjunto de boas práticas orientadas à gestão das organizações prestadoras de serviços (CMMI Product Team, 2010). O MR-MPS-SV é um modelo de referência para serviços (Softex, 2015). Comparando as normas e modelos, podemos identificar os principais requisitos e processos aplicáveis à Central de Serviços de TI.

\section{Trabalhos Relacionados}

Esta seção faz uma revisão de trabalhos que apresentam contribuições sobre gerenciamento de serviços de TI, melhoria da qualidade de processos em serviços de TI e implantação de Centrais de Serviços de TI. A Tabela 1 demonstra os trabalhos relacionados e o diferencial do trabalho proposto.

Tabela 1. Diferença do trabalho proposto em relação aos trabalhos anteriores

\begin{tabular}{|c|c|c|}
\hline \multicolumn{2}{|c|}{ Trabalhos Relacionados } & \multirow{2}{*}{ Diferencial do Trabalho Proposto } \\
\hline Autores & Título & \\
\hline $\begin{array}{l}\text { Araujo, et al. } \\
\text { (2014) }\end{array}$ & $\begin{array}{l}\text { Experiência de Implantação do } \\
\text { MR-MPS-SV no Service Desk da } \\
\text { ECO Sistemas }\end{array}$ & $\begin{array}{l}\text { Os autores aplicam um modelo de qualidade a uma Central de Serviços. } \\
\text { O trabalho proposto por esta pesquisa busca garantir a qualidade desde } \\
\text { o processo de implantação da Central de Serviços. }\end{array}$ \\
\hline $\begin{array}{l}\text { Tang e Todo } \\
\text { (2013) }\end{array}$ & $\begin{array}{l}\text { A Study of Service Desk Setup in } \\
\text { Implementing IT Service } \\
\text { Management in Enterprises }\end{array}$ & $\begin{array}{l}\text { O estudo de Tang e Todo foca na transformação de processos } \\
\text { específicos de uma Central de Serviços. Este trabalho propõe um } \\
\text { framework de implantação ou melhoria do serviço. }\end{array}$ \\
\hline $\begin{array}{l}\text { Rodrigues, Souza } \\
\text { e Oliveira (2013) }\end{array}$ & $\begin{array}{l}\text { Implementando o Nível G do MR- } \\
\text { MPS-SV com base no conceito de } \\
\text { Central de Serviços }\end{array}$ & $\begin{array}{l}\text { Os autores detalham os elementos de uma Central de Serviços e sua } \\
\text { relação com o modelo MPS. Esta pesquisa tem foco prático em "como } \\
\text { fazer" ao invés de "o que fazer". }\end{array}$ \\
\hline $\begin{array}{l}\text { Jantti, Cater-Steel } \\
\text { e Shrestha(2012) }\end{array}$ & $\begin{array}{l}\text { Towards an Improved IT Service } \\
\text { Desk System and Processes: A } \\
\text { Case Study }\end{array}$ & $\begin{array}{l}\text { Os autores analisam uma Central de Serviços em funcionamento e } \\
\text { propõem soluções aos desafios encontrados. Esta pesquisa propõe uma } \\
\text { solução para implantação ou melhoria de uma Central de Serviço. }\end{array}$ \\
\hline $\begin{array}{l}\text { Keller e } \\
\text { Midboe(2010) }\end{array}$ & $\begin{array}{l}\text { Implementing a service desk: A } \\
\text { practitioner's perspective }\end{array}$ & $\begin{array}{l}\text { Os autores focam em requisitos funcionais de processos específicos. O } \\
\text { trabalho proposto foca em um framework de implantação como um } \\
\text { todo, compreendendo desde a preparação até a operação. }\end{array}$ \\
\hline $\begin{array}{l}\text { Brigano e Barros } \\
(2010)\end{array}$ & $\begin{array}{l}\text { A Implantação de um Service } \\
\text { Desk: Um Estudo de Caso } \\
\text { Aplicando Conceitos do ITIL e do } \\
\text { PMBOK }\end{array}$ & $\begin{array}{l}\text { Os autores implantaram uma Central de Serviços com base nos } \\
\text { problemas operacionais. Este trabalho propõe uma solução com base em } \\
\text { melhores práticas. }\end{array}$ \\
\hline
\end{tabular}

Ao proceder à revisão bibliográfica em repositórios de teses e dissertações e em diversos periódicos, no período de 2010 a 2016, não foram encontrados trabalhos que propusessem um framework ou modelo com abordagem prática para a implantação e/ou melhoria de Centrais de Serviços, assim podemos classificar os trabalhos encontrados como moderadamente relacionados.

\section{O Framework QoS-TI}

O framework QoS-TI (Qualidade do serviço de suporte de TI) incorpora as melhores práticas identificadas nos modelos pesquisados, criando uma dinâmica de implantação e 
melhoria de centrais de serviços de TI, através de uma estrutura composta por um Ciclo de Vida e um Toolbox de processos, ver Figura 1.

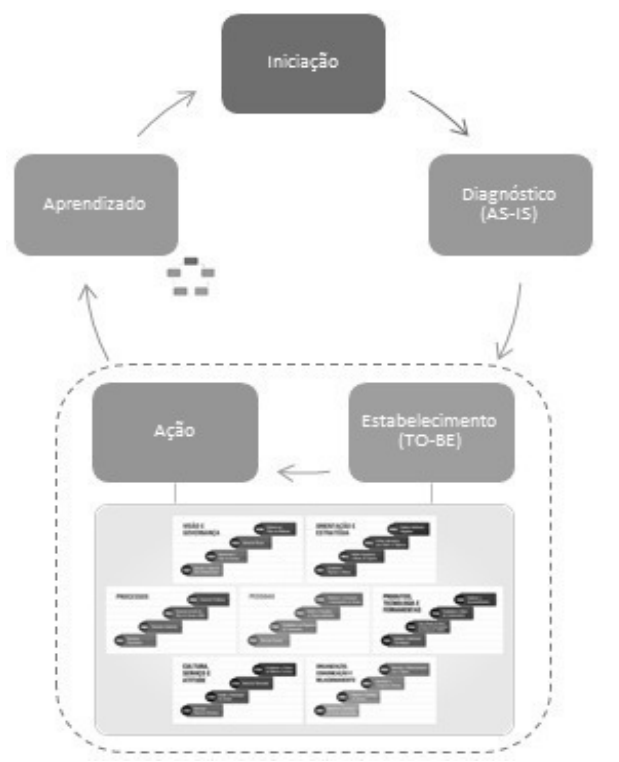

Figure 1. Estrutura do framework QoS-TI

O ciclo de vida consiste no conjunto de fases do projeto, estabelecendo o que precisa ser feito para alcançar os objetivos (Pmbok, 2013). Este ciclo de vida está detalhado na subseção 4.1. Já o Toolbox, consiste em um conjunto de dimensões que fornecem processos com abordagem prática para apoiar a implantação e/ou melhoria de centrais de serviços. Este Toolbox está detalhado na subseção 4.2.

Para facilitar o acesso pelos potenciais usuários, todos os fluxos e templates, utilizados nas fases do ciclo de vida e os processos do Toolbox foram disponibilizados no seguinte endereço eletrônico: https://sites.google.com/site/frameworkqosti/.

O framework foi originado atendendo os princípios descritos a seguir:

- Adaptabilidade - capacidade de aplicação a novas centrais de serviços ou às já existentes (o framework é preparado para implantar uma central de serviços do "zero" ou para diagnosticar problemas no serviço e implementar melhorias);

- Entrega de resultado - adota como estratégia de implantação uma abordagem sequencial/iterativa, permitindo agregar valor ao cliente no decurso da execução do projeto, através das fases e da implantação dos processos;

- Flexibilidade - pode ser utilizado para que a própria organização realize a implantação da Central de Serviços de TI ou como parâmetro para terceirização;

- Foco na qualidade - incorpora práticas existentes em modelos especializados para o gerenciamento de processos e serviços;

- Praticidade - descreve como implantar e/ou melhorar uma Central de Serviços de TI, através de uma abordagem prática, que é composta por um ciclo de vida e um Toolbox de processos. 


\subsection{Ciclo de Vida}

A implantação do framework QoS-TI é realizada através de diversas fases e esse conjunto de fases é denominado Ciclo de Vida. O ciclo de vida do framework QoS-TI é composto por cinco fases, adaptadas do modelo IDEAL (MCFEELEY, 1996): iniciação; diagnóstico; estabelecimento; ação e aprendizado.

O ciclo de vida inicia com a identificação das razões para a mudança e a construção do patrocínio para o projeto. Em seguida é realizado um diagnóstico da situação atual (AS-IS) para identificação de pontos de melhoria. Logo após, são definidos os objetivos e o plano de ações para alcançar a situação desejada (TO-BE). A fase Ação orienta a organização a implementar as ações planejadas, já a fase Aprendizado completa o ciclo com o objetivo de aperfeiçoar o serviço prestado.

Uma fase engloba um conjunto de atividades, relacionadas de maneira lógica, onde a sua conclusão é marcada por entregas (Pmbok, 2013). As fases possuem fluxos, atividades e templates. As fases do framework QoS-TI são apresentadas a seguir.

Fase Iniciação: A fase Iniciação representa o início do ciclo de vida do framework, seu propósito é construir uma base para a oferta do serviço. Para isso, a fase busca responder às seguintes perguntas: As razões da organização para empreender o esforço estão claramente definidas? As partes interessadas estão identificadas? Qual a estratégia de oferta do serviço? Existe infraestrutura para gerenciar a execução do projeto de implantação ou melhoria? O fluxo que representa a fase Iniciação é apresentado na Figura 2.

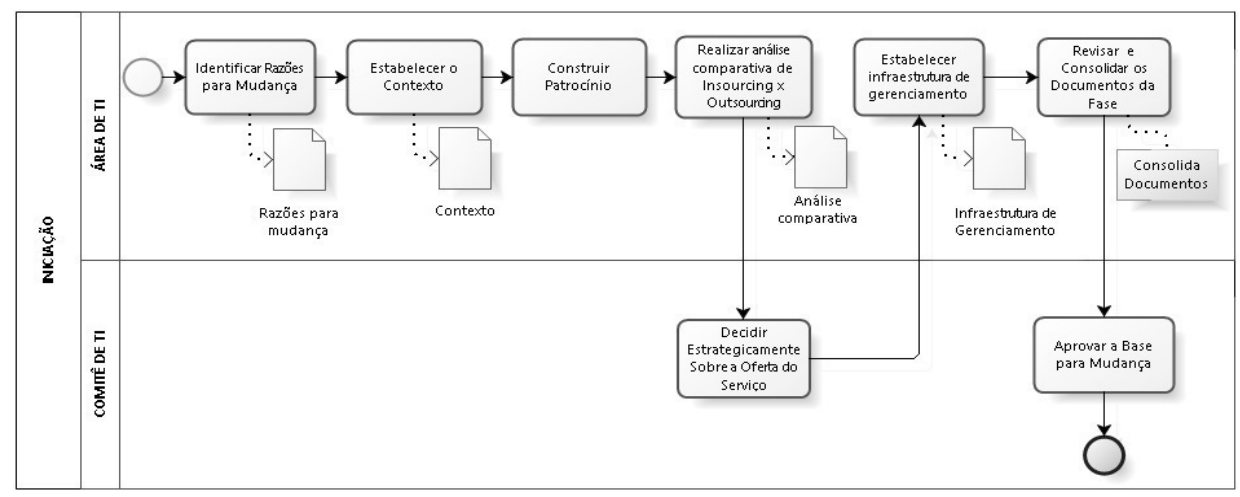

Figura 2: Fluxo da fase Iniciação do framework QoS-TI

O fluxo começa com a identificação das razões para realizar a mudança organizacional. Em seguida, busca-se estabelecer o contexto, ter clareza sobre a visão organizacional e do que ela significa para a área de TI. A atividade "construir patrocínio" busca o comprometimento dos stakeholders com os recursos essenciais para o projeto. A análise comparativa de insourcing x outsourcing visa subsidiar a decisão da oferta do serviço, se é mais vantajoso subcontratar ou oferecer com o quadro de pessoal próprio. Após a análise comparativa, o Comitê Gestor de TI avalia e discute as possibilidades, e decide qual a estratégia de oferta do serviço será adotada. Por último, a atividade "estabelecer a infraestrutura de gerenciamento" define a equipe de gerenciamento do trabalho, o cronograma inicial e o estabelecimento de regras de comunicação. Os templates da fase Iniciação são: razões para mudança; contexto; análise comparativa; infraestrutura de gerenciamento. 
Fase Diagnóstico: O propósito da fase Diagnóstico é desenvolver um entendimento sobre a situação atual (AS-IS), a fim de identificar necessidades de melhoria e desenvolver recomendações. Nesta fase procuramos responder à seguinte pergunta: Como estamos agora?

O instrumento de diagnóstico é um questionário, disponível nos templates do framework. O resultado do diagnóstico identifica necessidades e oportunidades de melhoria na oferta do serviço de suporte de TI. O principal artefato produzido nesta fase é o relatório de resultados e recomendações. $\mathrm{O}$ fluxo que representa a fase Diagnóstico é apresentado na Figura 3.

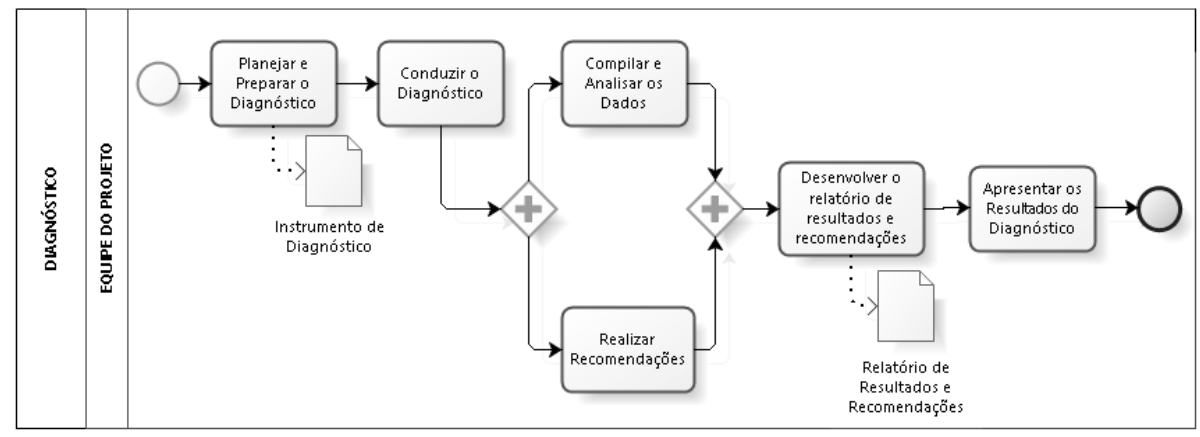

Figura 3: Fluxo da fase Diagnóstico do framework QoS-TI

O fluxo inicia com o planejamento do diagnóstico, são definidos: o período, o local, os respondentes, o tempo, os critérios e a forma de aplicação do diagnóstico. Nessa etapa é importante que o objetivo do diagnóstico seja totalmente entendido pelos respondentes, os quais devem ter características e conhecimentos suficientes para que as respostas sejam as mais assertivas possíveis. Sugerimos reunir a equipe e aplicar o diagnóstico, abrindo espaço para discussões e a obtenção de uma visão homogênea da área de TI sobre os itens avaliados. Após a aplicação do diagnóstico e a compilação dos resultados, a atividade "realizar recomendações" aponta os pontos fortes, pontos fracos e as recomendações de melhoria. Por fim, a apresentação dos resultados do diagnóstico às partes interessadas visa demonstrar a relevância e justificar futuras decisões, mantendo o patrocínio e o apoio da organização. Os templates da fase Diagnóstico são: instrumento de diagnóstico; resultados do diagnóstico e recomendações.

Fase Estabelecimento: A fase Estabelecimento realiza a definição dos objetivos e o planejamento da situação desejada (TO-BE), procurando responder às seguintes perguntas: Onde desejamos chegar? Qual a central de serviços de TI que queremos ter?

Para responder a estas perguntas serão realizadas duas importantes atividades: a identificação de oportunidades de melhoria e a seleção de objetivos. As oportunidades de melhoria são identificadas com base nos resultados do diagnóstico, e a seleção de objetivos através da escolha, no Toolbox, de processos que iremos implantar. A visão resumida do Toolbox está detalhada na seção 4.4. O fluxo que representa a fase Estabelecimento pode ser visualizado na Figura 4. 


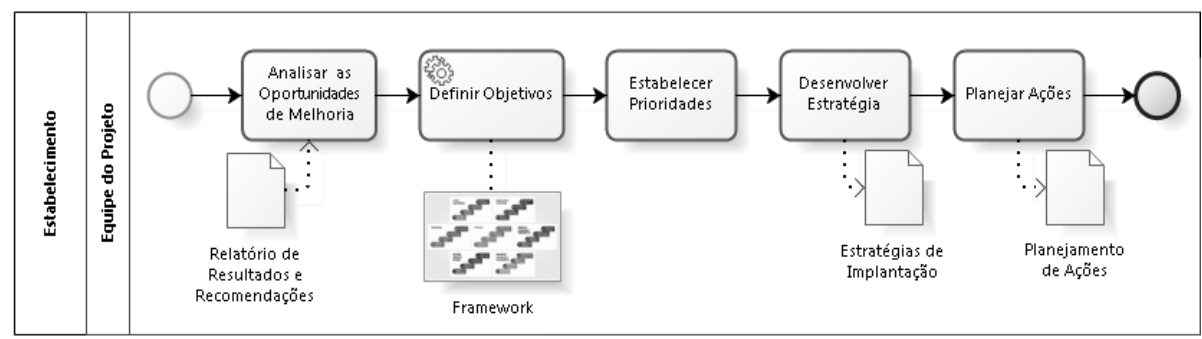

Figura 4: Fluxo da fase Estabelecimento do framework QoS-TI

O fluxo inicia com a análise das oportunidades de melhoria do serviço. Em seguida, o Toolbox de processos é utilizado para a definição de objetivos, ou seja, a escolha de quais dimensões pretende-se abordar e quais processos se quer implantar. A seleção dos objetivos define a situação desejada (TO-BE) e os processos que serão executados na fase Ação. Deve-se considerar a limitação de recursos, as dependências existentes entre as atividades, fatores externos que podem intervir nas mudanças e as prioridades da organização. Estratégias precisam ser desenvolvidas para preparar a organização e a área de TI para a implantação da solução, e para que o serviço entre em operação. As estratégias a serem definidas incluem fatores de ordem técnica e não técnica, incluindo a cultura da organização, aspectos políticos, prováveis fontes de resistência, limitação de recursos, excesso de atividades, dentre outras dificuldades. Por último, um planejamento de ações deve ser desenvolvido, incluindo tarefas, pontos de decisão, recursos, responsabilidades, métricas, mecanismos de controle de riscos e outros elementos requeridos pela organização. Os templates da fase Estabelecimento são: estratégias de implantação; e planejamento de ações.

Fase Ação: O propósito da fase Ação é apoiar a organização na implementação da iniciativa que foi planejada nas fases anteriores. A fase Ação procura responder à seguinte questão: Como chegaremos lá? Para isso, são executadas atividades de criação, validação, teste, refinamento e implementação da solução (estratégias, ações planejadas e processos selecionados). A Figura 5 apresenta o fluxo que representa a fase Ação.

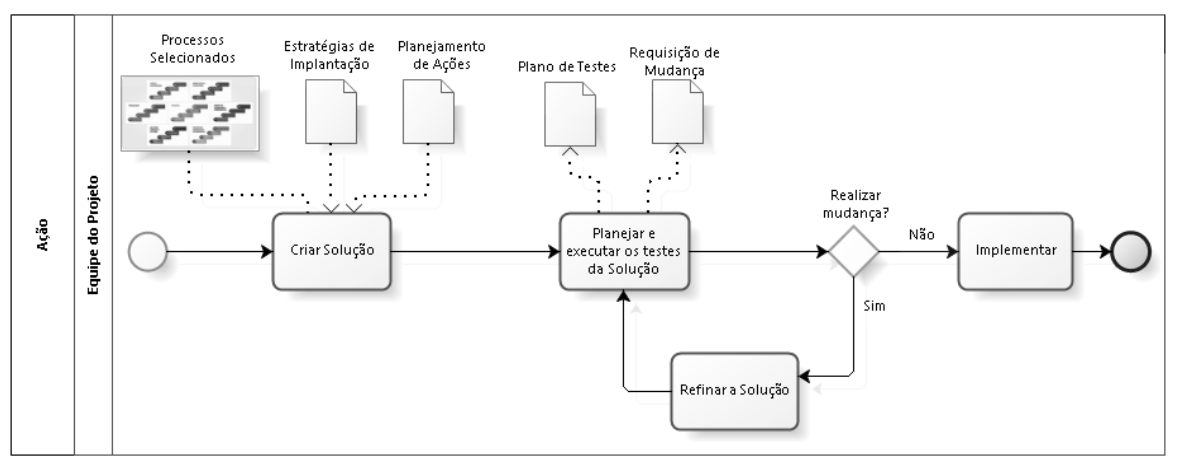

Figura 5: Fluxo da fase Ação do framework QoS-TI

A equipe do projeto realiza a validação da solução criada na fase Estabelecimento, podendo acrescentar o uso de ferramentas, técnicas e outros conhecimentos e habilidades. Serão realizados os ajustes necessários, visando obter a "melhor solução" para a entrada em operação. A solução precisa ser testada para verificar se funciona conforme o planejado. Será realizado um teste, no qual é verificado o funcionamento do sistema, dos processos, a coordenação da equipe, a interação com 
os usuários, dentre outros. As falhas devem ser identificadas e corrigidas, refletindo o conhecimento, experiência e as lições aprendidas. Várias iterações do processo de testar e refinar podem ser necessárias para o alcance de uma solução satisfatória. Por fim, a solução pode ser implementada na organização, e para isso será utilizada a abordagem definida na estratégia da fase Estabelecimento. Nesta etapa, os processos do Toolbox que foram escolhidos serão implementados. Os templates da fase Ação são: estratégias de implantação (produzido na fase Estabelecimento); planejamento de Ações (produzido na fase Estabelecimento); plano de testes; e requisição de mudança.

Fase Aprendizado: O propósito da fase Aprendizado é continuar a aprimorar a implementação de mudanças. A experiência na utilização do framework QoS-TI é revisada para verificar o que foi alcançado, se o esforço atingiu os objetivos definidos, e como a organização pode implementar futuras mudanças de maneira mais efetiva e eficiente. Os registros devem ser mantidos ao longo da aplicação do ciclo de vida do framework, tendo em vista a oportunidade de aprendizado e melhoria do processo de implantação. A fase procura responder às seguintes perguntas: Conseguimos chegar? $\mathrm{O}$ que aprendemos? O fluxo que representa a fase Aprendizado, Figura 6, é apresentado a seguir.

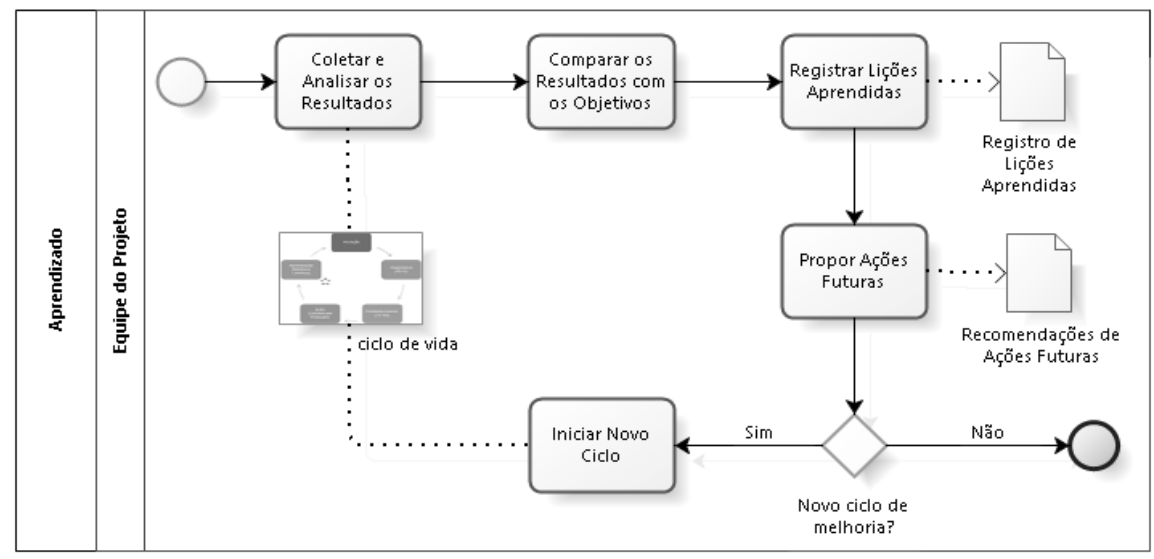

Figura 6: Fluxo da fase Aprendizado do framework QoS-TI

O fluxo inicia com a coleta e análise dos resultados obtidos durante todo o ciclo de vida do framework. Em seguida, é feita a comparação dos resultados obtidos para verificar o que foi alcançado com o esforço realizado. Faz-se o registro das lições aprendidas durante todo ciclo de vida, com a finalidade de realizar aperfeiçoamentos para experiências futuras. Recomendações para melhorias futuras são desenvolvidas e documentadas com base nos resultados e nas lições aprendidas. Caso necessário, iniciase um novo ciclo de vida em resposta às recomendações de melhorias futuras. Os templates da fase aprendizado são: registro de lições aprendidas; e recomendações de ações futuras.

\subsection{Toolbox de Processos}

O objetivo do Toolbox é apoiar a implantação e melhoria de centrais de serviços de TI durante a execução das fases Estabelecimento e Ação do ciclo de vida. Para isso, possui uma abordagem prática, apresentando vários processos distribuídos em uma estrutura composta por dimensões. O Toolbox foi elaborado com base nos seguintes conceitos: estrutura de melhoria baseada nas dimensões do Extended Process Maturity 
Framework-EPMF (Rudd, 2010); estrutura de processos que apoiam a implantação das fases do ciclo de vida; compatibilidade com a abordagem iterativa e incremental (Krutchen, 2003).

O principal motivador para o desenvolvimento do Toolbox de processos foi a dificuldade de encontrar orientações práticas que auxiliem a implantação e melhoria de centrais de serviços de TI. A sua aplicação foi detalhada nas fases Estabelecimento e Ação do ciclo de vida, apresentadas na seção 4.1. O Toolbox é composto por dimensões e processos, conforme pode ser observado na Tabela 2.

Tabela 2. Dimensões, processos e objetivos do Toolbox

\begin{tabular}{|c|c|c|}
\hline Dimensões & Processos & Objetivos \\
\hline \multirow{4}{*}{$\begin{array}{l}\text { Visão e } \\
\text { Governança }\end{array}$} & $\begin{array}{l}\text { VG04 - Elaborar um Plano de } \\
\text { Melhoria }\end{array}$ & $\begin{array}{l}\text { Construir um plano de melhoria contínua, constituído por um conjunto de metas } \\
\text { e ações estabelecidas a partir de resultados obtidos com o processo de } \\
\text { autoavaliação do serviço de suporte de TI. }\end{array}$ \\
\hline & VG03 - Gerenciar Riscos & $\begin{array}{l}\text { Aumentar a probabilidade de alcance dos objetivos da organização, reduzindo } \\
\text { os riscos a níveis aceitáveis. }\end{array}$ \\
\hline & $\begin{array}{l}\text { VG02 - Demonstrar o Valor do } \\
\text { Serviço }\end{array}$ & Demonstrar o valor do serviço para a organização. \\
\hline & $\begin{array}{l}\text { VG01 - Garantir o Apoio da Alta } \\
\text { Administração }\end{array}$ & $\begin{array}{l}\text { Garantir o apoio dos integrantes da alta direção na implantação e manutenção } \\
\text { da Central de Serviços de TI. }\end{array}$ \\
\hline \multirow{4}{*}{$\begin{array}{l}\text { Orientação e } \\
\text { Estratégia }\end{array}$} & $\begin{array}{l}\text { OE04 - Realizar Auditorias } \\
\text { Regulares }\end{array}$ & $\begin{array}{l}\text { Estabelecer revisões ou auditorias internas para verificar a eficiência, eficácia e } \\
\text { o cumprimento das atividades de melhoria. }\end{array}$ \\
\hline & $\begin{array}{l}\text { OE03 - Definir Indicadores para } \\
\text { Medir os Objetivos }\end{array}$ & Definir indicadores de desempenho para medir os objetivos estabelecidos. \\
\hline & $\begin{array}{l}\text { OE02 - Definir Arquitetura e } \\
\text { Níveis de Suporte }\end{array}$ & $\begin{array}{l}\text { Definir a arquitetura da Central de Serviços e os níveis de atendimento ao } \\
\text { usuário. }\end{array}$ \\
\hline & $\begin{array}{l}\text { OE01 - Estabelecer Objetivos e } \\
\text { Metas }\end{array}$ & $\begin{array}{l}\text { Estabelecer objetivos para a Central de Serviços de TI, com metas formalmente } \\
\text { definidas. }\end{array}$ \\
\hline \multirow{4}{*}{ Processos } & PR04 - Gerenciar Problema & $\begin{array}{l}\text { Minimizar o impacto adverso de incidentes e problemas decorrentes de erros } \\
\text { conhecidos relacionados com a infraestrutura de TI e prevenir a reincidência } \\
\text { desses erros. }\end{array}$ \\
\hline & $\begin{array}{l}\text { PR03 - Gerenciar Acordo de Nível } \\
\text { de Serviço (ANS) }\end{array}$ & $\begin{array}{l}\text { Garantir que todos os serviços de suporte de TI sejam entregues dentro de metas } \\
\text { atingíveis acordadas, fomentando medidas corretivas quando necessário. }\end{array}$ \\
\hline & PR02 - Gerenciar Incidentes & $\begin{array}{l}\text { Restaurar a operação normal do serviço o mais rápido possível e minimizar o } \\
\text { impacto adverso sobre as operações da organização. }\end{array}$ \\
\hline & PR01－Gerenciar Requisições & $\begin{array}{l}\text { Manter a satisfação do usuário através do processo de gerenciamento do ciclo } \\
\text { de vida de todas as requisições de serviço realizadas. }\end{array}$ \\
\hline \multirow{4}{*}{ Pessoas } & $\begin{array}{l}\text { PS04 - Monitorar e Comunicar o } \\
\text { Desempenho do Serviço }\end{array}$ & $\begin{array}{l}\text { Monitorar e comunicar a execução e o desempenho do serviço às partes } \\
\text { interessadas para apoiar a tomada de decisão. }\end{array}$ \\
\hline & $\begin{array}{l}\text { PS03 - Definir e Formalizar as } \\
\text { Responsabilidades }\end{array}$ & $\begin{array}{l}\text { Definir e descrever os papéis e responsabilidades dos profissionais da Central } \\
\text { de Serviços de TI. }\end{array}$ \\
\hline & $\begin{array}{l}\text { PS02 - Estabelecer um Programa } \\
\text { de Treinamento }\end{array}$ & $\begin{array}{l}\text { Estabelecer um programa de treinamento para aperfeiçoar as habilidades e } \\
\text { competências da equipe da Central de Serviços. }\end{array}$ \\
\hline & PS01 - Recrutar Pessoal & $\begin{array}{l}\text { Selecionar profissionais com habilidades e capacidades adequadas ao } \\
\text { cumprimento das funções da Central de Serviços. }\end{array}$ \\
\hline \multirow{4}{*}{$\begin{array}{l}\text { Produtos, } \\
\text { Tecnologia e } \\
\text { Ferramentas }\end{array}$} & $\begin{array}{l}\text { TC04 - Implantar o } \\
\text { Autoatendimento }\end{array}$ & $\begin{array}{l}\text { Permitir que o usuário busque soluções e orientações por conta própria, a fim de } \\
\text { aumentar o acesso à informação e diminuir o tempo de resolução de problemas. }\end{array}$ \\
\hline & $\begin{array}{l}\text { TC03 - Estabelecer a Base de } \\
\text { Conhecimento }\end{array}$ & $\begin{array}{l}\text { Permitir ao provedor de serviços ser mais eficiente, melhorar a qualidade do } \\
\text { serviço e aumentar a satisfação do cliente por meio da redução da necessidade } \\
\text { de redescobrir conhecimento. }\end{array}$ \\
\hline & $\begin{array}{l}\text { TC02 - Criar e Manter um Banco } \\
\text { de Dados de Gerenciamento de } \\
\text { Configuração (CMDB) }\end{array}$ & $\begin{array}{l}\text { Garantir que a informação precisa e confiável sobre os ativos de serviço esteja } \\
\text { disponível quando necessária. }\end{array}$ \\
\hline & $\begin{array}{l}\text { TC01 - Analisar e Selecionar } \\
\text { Tecnologias }\end{array}$ & $\begin{array}{l}\text { Analisar e selecionar tecnologias de apoio aos processos e à operação de } \\
\text { serviços. }\end{array}$ \\
\hline
\end{tabular}




\begin{tabular}{|c|c|c|}
\hline Dimensões & Processos & Objetivos \\
\hline \multirow{4}{*}{$\begin{array}{l}\text { Cultura, Serviço } \\
\text { e Atitude }\end{array}$} & $\begin{array}{l}\text { CT04 - Estabelecer a Cultura de } \\
\text { Melhoria Contínua }\end{array}$ & $\begin{array}{l}\text { Disseminar premissas e práticas de melhoria contínua na Central de Serviços de } \\
\text { TI. }\end{array}$ \\
\hline & CT03 - Gerenciar Demandas & $\begin{array}{l}\text { Obter o entendimento da demanda exigida e garantir que a Central de Serviços } \\
\text { tenha capacidade suficiente para atendê-la. }\end{array}$ \\
\hline & $\begin{array}{l}\text { CT02 - Avaliar a Satisfação do } \\
\text { Usuário }\end{array}$ & $\begin{array}{l}\text { Avaliar o serviço prestado, conforme a percepção do usuário, permitindo a } \\
\text { identificação de possíveis falhas para que melhorias sejam realizadas. }\end{array}$ \\
\hline & $\begin{array}{l}\text { CT01 - Gerenciar Recursos } \\
\text { Humanos }\end{array}$ & $\begin{array}{l}\text { Melhorar os resultados da Central de Serviços de TI através do gerenciamento } \\
\text { do recurso humano. }\end{array}$ \\
\hline \multirow{4}{*}{$\begin{array}{l}\text { Organização, } \\
\text { Comunicação e } \\
\text { Relacionamento }\end{array}$} & $\begin{array}{l}\text { OR04 - Gerenciar o } \\
\text { Relacionamento com o Cliente }\end{array}$ & $\begin{array}{l}\text { Manter um relacionamento positivo com os clientes, em torno do atendimento } \\
\text { dos requisitos organizacionais. }\end{array}$ \\
\hline & $\begin{array}{l}\text { OR03 - Identificar e Categorizar } \\
\text { Clientes }\end{array}$ & $\begin{array}{l}\text { Identificar e categorizar os clientes de acordo com a criticidade dos serviços } \\
\text { oferecidos, a fim de orientar a priorização do atendimento. }\end{array}$ \\
\hline & $\begin{array}{l}\text { OR02 - Elaborar o Catálogo de } \\
\text { Serviços }\end{array}$ & $\begin{array}{l}\text { Criar e manter um Catálogo de Serviços com informações sobre todos os } \\
\text { serviços de TI em produção e aqueles disponíveis para implantação. }\end{array}$ \\
\hline & $\begin{array}{l}\text { OR01 - Identificar e Gerenciar as } \\
\text { Partes Interessadas }\end{array}$ & $\begin{array}{l}\text { Tem como objetivo identificar, qualificar e gerenciar as partes interessadas da } \\
\text { Central de Serviços de TI. }\end{array}$ \\
\hline
\end{tabular}

O Toolbox contém 28 processos $^{1}$ distribuídos entre sete dimensões. Cada processo possui: objetivo, responsável, entradas, tarefas, técnicas e ferramentas, métricas e indicadores, saídas e referências. Eventualmente, podem ser apresentados detalhamentos adicionais, como fluxos ou estratégias. Os processos do Toolbox incorporam diversas práticas de gerenciamento de serviços de TI e gestão da qualidade, as normas e modelos estão referenciadas em cada processo.

\section{Avaliação do Framework QoS-TI}

Esta seção descreve a avaliação do framework QoS-TI por especialistas dos Institutos Federais. O procedimento de avaliação compreendeu as seguintes etapas: definição do método de avaliação; teste piloto; execução da avaliação; análise e apresentação de resultados.

O instrumento de avaliação foi a aplicação de um questionário através de um survey, desenvolvido para obter a percepção dos especialistas em relação aos seguintes aspectos do framework: clareza, foco na qualidade, utilidade, adaptabilidade, entrega de resultado, adequação e abrangência, e flexibilidade. O questionário foi formado por dois blocos, com dez itens no total: o bloco I capturou o perfil do respondente; o bloco II foi composto por perguntas que medem o nível de concordância ou não em relação aos aspectos do framework, através da escolha de uma das seguintes opções da escala de Likert: discordo totalmente; discordo em parte; não concordo, nem discordo; concordo em parte ou concordo totalmente. A opinião "não sei responder" foi disponibilizada apenas para a questão três, pois exigia do respondente o conhecimento prévio sobre normas e modelos de gerenciamento de serviços.

A Tabela 3 demonstra os aspectos avaliados e suas respectivas questões. Para a criação das questões levamos em consideração algumas características de modelos de gerenciamento de serviços de TI (foco na qualidade, entrega de resultados) e os princípios do framework.

1 O conteúdo dos processos do Toolbox está disponível no seguinte endereço eletrônico: https://sites.google.com/site/frameworkqosti/toolbox-de-processos/estrutura. 
Tabela 3. Aspectos avaliados no framework e suas respectivas questões

\begin{tabular}{|c|c|l|}
\hline Aspectos & \multicolumn{2}{|c|}{ Questões } \\
\hline \multirow{2}{*}{ Clareza } & Q1 & As atividades e os artefatos de cada fase do ciclo de vida estão claros e compreensíveis? \\
\cline { 2 - 4 } & Q2 & A arquitetura do Toolbox, composta por dimensões e processos, está clara e compreensível? \\
\hline $\begin{array}{c}\text { Foco na } \\
\text { Qualidade }\end{array}$ & Q3 & $\begin{array}{l}\text { O framework QoS-TI pode ser utilizado em conjunto com as melhores práticas de gerenciamento de } \\
\text { serviços de TI (ITIL, ISO/IEC 20.000, CMMI for services, MR-MPS serviços, dentre outras)? }\end{array}$ \\
\hline Utilidade & Q4 & $\begin{array}{l}\text { A estrutura apresentada pelo framework QoS-TI facilita a implantação ou a melhoria de uma Central } \\
\text { de Serviços de TI? }\end{array}$ \\
\hline Adaptabilidade & Q5 & $\begin{array}{l}\text { O framework QoS-TI é adaptável, ou seja, pode ser utilizado para implantar uma central de serviços } \\
\text { de TI do "zero" ou implementar melhorias em uma já existente? }\end{array}$ \\
\hline $\begin{array}{c}\text { Entrega de } \\
\text { Resultado }\end{array}$ & Q6 & $\begin{array}{l}\text { O framework QoS-TI permite agregar valor ao cliente no decurso da realização do projeto, através } \\
\text { da execução das fases e dos processos? }\end{array}$ \\
\hline \multirow{2}{*}{$\begin{array}{c}\text { Adequação e } \\
\text { Abrangência }\end{array}$} & Q7 & $\begin{array}{l}\text { O framework QoS-TI pode ser aplicado para melhorar a qualidade do serviço de suporte de TI na } \\
\text { instituição onde trabalho? }\end{array}$ \\
\cline { 2 - 3 } & Q8 & $\begin{array}{l}\text { Recomendaria o framework QoS-TI para outras instituições que necessitam implantar uma Central } \\
\text { de Serviços de TI? }\end{array}$ \\
\hline Flexibilidade & Q9 & $\begin{array}{l}\text { Utilizaria o framework QoS-TI como apoio ao processo de contratação, no caso de uma decisão } \\
\text { estratégica pela terceirização do serviço de operação de uma central de serviços de TI? }\end{array}$ \\
\hline
\end{tabular}

O framework ficou disponível para análise dos especialistas no seguinte endereço eletrônico: https://sites.google.com/site/frameworkqosti/.

Antes da aplicação definitiva da avaliação foi realizado um teste-piloto. O teste foi concebido para permitir um processo gradual de aperfeiçoamento do questionário. Pelo caráter experimental, o teste foi aplicado a uma amostra de oito profissionais com experiência no suporte de TI. Foram avaliados os seguintes aspectos: orientações, clareza das perguntas, o tempo necessário para conhecer o framework e responder o questionário, estrutura e organização, diagramação e pertinência.

A estratégia adotada para a avaliação do framework foi o envio do convite para a lista de e-mails do Fórum de TI dos IFs - FORTI, dos quais os diretores de TI fazem parte. Além disso, a pesquisa foi divulgada à comunidade de profissionais de TI dos IFs no grupo gsti-if@googlegroups.com. A participação dos especialistas envolveu as seguintes etapas: acessar e conhecer o framework QoS-TI, responder o questionário. Foi disponibilizado contato para que os especialistas pudessem esclarecer dúvidas sobre o funcionamento do framework. Foram realizadas 51 avaliações, no período de 10 a 19 de maio de 2017, por especialistas distribuídos em 19 Institutos Federais, o que representou $50 \%$ do total de IFs.

\subsection{Resultados da Avaliação com Especialistas}

Os especialistas são profissionais que trabalham nos Institutos Federais e possuem experiência com o serviço de suporte de TI. Foi observado que $82 \%$ dos especialistas possuem mais de três anos de experiência com o serviço de suporte de TI, $12 \%$ possuem de um a três anos, e apenas $6 \%$ possuem até um ano de experiência.

Podemos observar na Figura 7, que a maioria dos especialistas concordou totalmente sobre os aspectos do framework avaliados, o que correspondeu a $59 \%$ das respostas. Um percentual significativo concordou em parte, representando $32 \%$ das respostas, 7\% das respostas relataram a opinião "não concordo, nem discordo" e apenas $1 \%$ das respostas assinalaram a opção "discordo em parte", não tivemos nenhuma resposta "discordo totalmente". Diante dessa avaliação, entendemos que o framework 
foi muito bem conceituado pelos especialistas. A seguir apresenta-se o detalhamento da avaliação por questão.

A respeito do aspecto "clareza" das atividades e artefatos das fases do ciclo de vida, $47 \%$ dos especialistas concordam totalmente, outros $47 \%$ concordam em parte, $4 \%$ não concordam e nem discordam, e apenas $2 \%$ discordaram parcialmente, ver Figura 8.

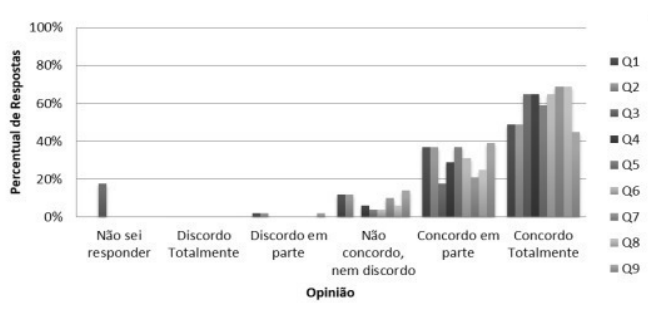

Figura 7: Percentual de respostas por Opinião

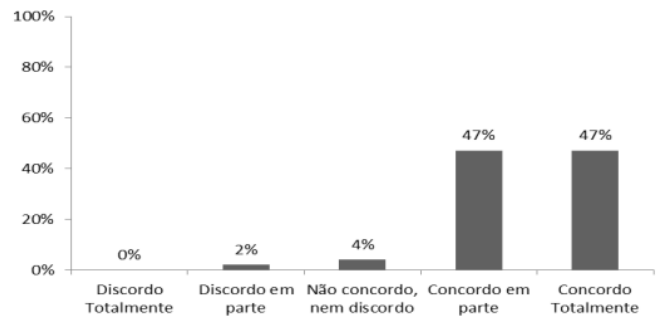

Figura 8: Resultados sobre a clareza das atividades e artefatos

Quando se trata da clareza do Toolbox de processos o percentual de especialistas que concordaram totalmente sobe para $49 \%$, seguido de $37 \%$ que concordaram em parte, ver Figura 9. Com relação ao "foco na qualidade", podemos observar na Figura 10 que $64,70 \%$ dos especialistas concordaram totalmente sobre a possibilidade do framework ser utilizado em conjunto com outras normas e modelos de qualidade, o que evidencia também a característica de compatibilidade.

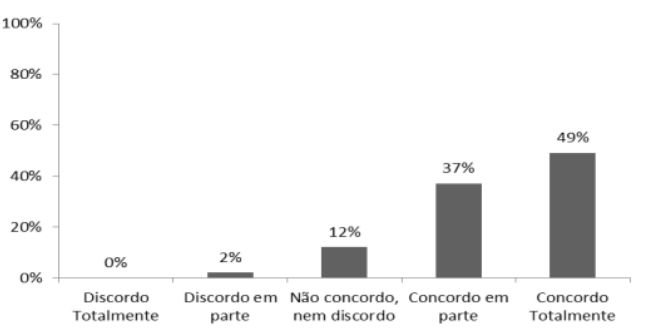

Figura 9: Resultados sobre a clareza do Toolbox

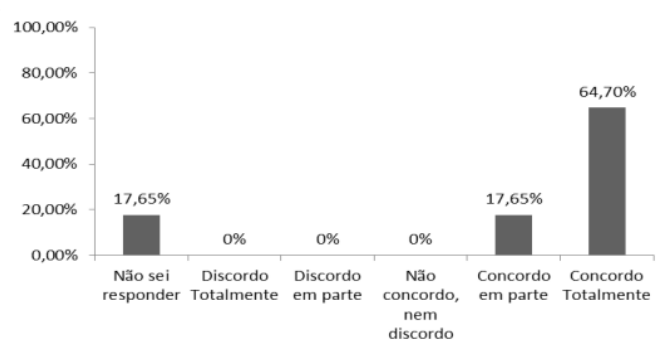

Figura 10: Resultados acerca do foco na qualidade

Sobre o aspecto "utilidade", $65 \%$ dos especialistas acreditam que o framework facilita a implantação ou melhoria de Centrais de Serviços, o que demonstra uma relevante indicação dos benefícios do framework, ver Figura 11. Acerca do aspecto "adaptabilidade", predominou a opinião de que o framework pode ser utilizado para implantar uma Central de Serviços do "zero" ou também para implementar melhorias em uma Central já em operação, sendo que 59\% dos especialistas concordam totalmente, e 37\% concordam em parte, ver Figura 12.

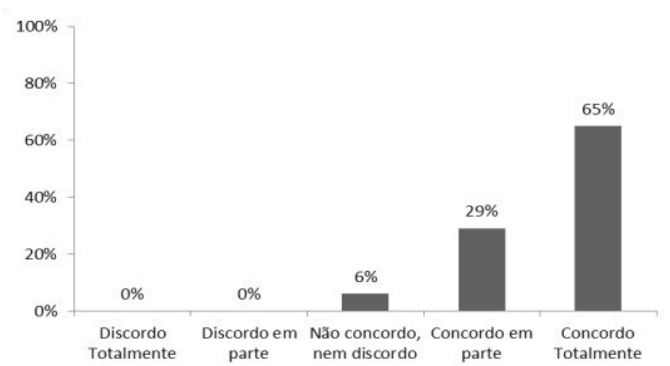

Figura 11: Resultados em relação à utilidade

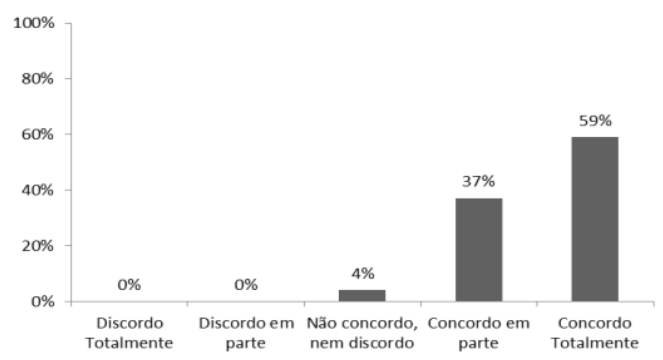

Figura 12: Resultados acerca da adaptabilidade 
Podemos observar na Figura 13 que os resultados da avaliação reforçam um dos princípios do framework a "entrega de resultado", pois $65 \%$ dos especialistas concordam totalmente sobre a agregação de valor ao cliente no decurso da realização do projeto, e $31 \%$ concordam em parte. $\mathrm{O}$ aspecto "adequação e abrangência" foi o melhor avaliado entre os especialistas, $69 \%$ concordaram totalmente sobre a possibilidade de o framework ser aplicado para melhorar a qualidade do serviço de suporte de TI na instituição onde trabalha, ver Figura 14.

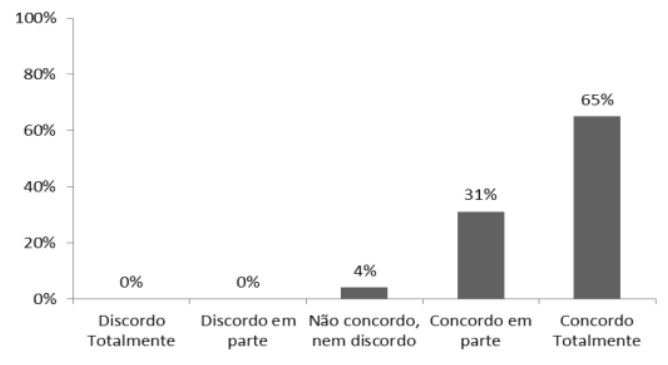

Figura 13: Resultados em relação à entrega de resultados

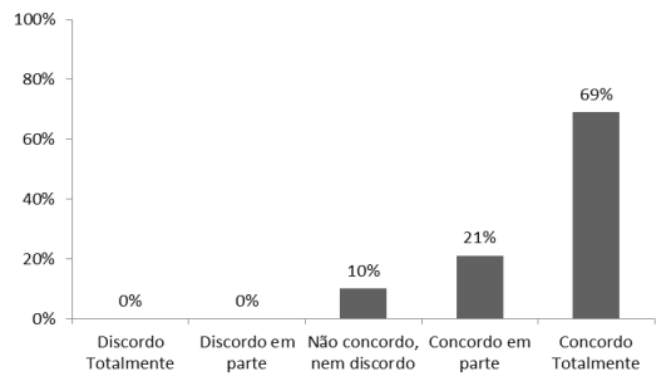

Figura 14: Resultados sobre a adequação

Ainda sobre o aspecto adequação e abrangência, 69\% dos respondentes afirmaram que concordam totalmente sobre a recomendação do framework para outras instituições que necessitam implantar uma Central de Serviços, e 25\% concordam em parte, ver Figura 15. Sobre a utilização do framework como apoio ao processo de contratação, no caso de uma decisão estratégica pela terceirização, $45 \%$ dos especialistas opinaram concordo totalmente e 39\% concordo em parte, ver Figura 16. Destacamos o percentual de opiniões "não concordo, nem discordo", o que é justificável, pois o framework oferece apoio ao processo de contratação, todavia tem o foco no processo de implantação e melhoria.

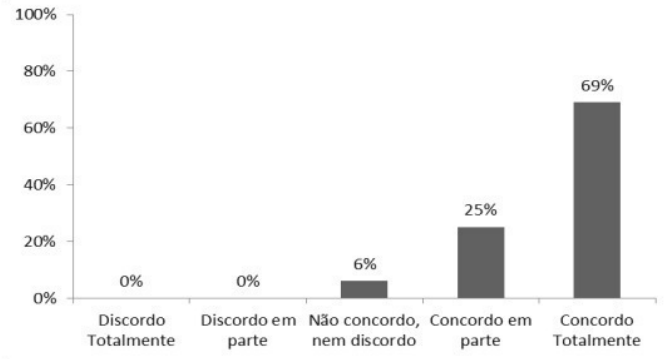

Figura 15: Resultados acerca da abrangência

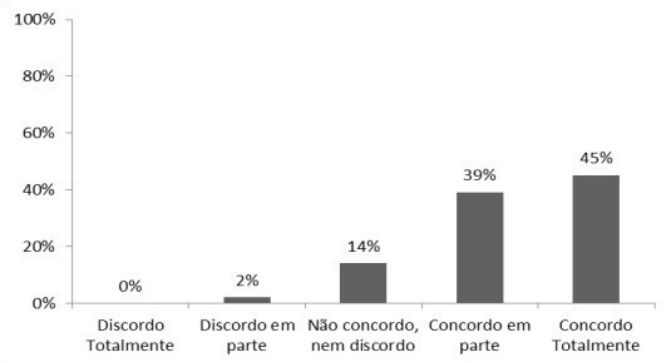

Figura 16: Resultados sobre a utilização do framework no caso de terceirização

Em relação aos pontos fortes do framework, os especialistas relataram: ciclo de vida bem definido; organização e clareza; incorpora conceitos de modelos de referência amplamente utilizados no mercado; adaptável e flexível; foco prático; simplicidade; facilidade de leitura e compreensão; possibilita ao gestor definir prioridades; Toolbox de processos com abordagem prática; foco na qualidade e satisfação dos usuários; conceito de melhoria contínua; adequado à realidade dos IFs; integração com outros modelos; disponibiliza templates; possibilidade real de utilização; e apresenta uma proposta que pode gerar impactos positivos e significativos para a estratégia organizacional.

Os especialistas ainda fizeram algumas observações, dentre as quais destacamos: desenvolver uma versão resumida; incluir o processo gerenciar mudanças; necessidade 
de detalhamento dos papéis e responsabilidades; criação de um mapa mental para representar todo framework; a criação de vídeo aulas sobre a utilização do framework. Concluiu-se que os resultados foram satisfatórios e que a avaliação do framework QoSTI, por especialistas, responde à questão de pesquisa definida na introdução desse trabalho.

\section{Considerações Finais}

Após a realização da revisão da literatura, onde foram levantadas as melhores práticas para serviços de TI, apresentamos neste trabalho o framework QoS-TI como proposta para implantação e/ou melhoria de uma central de serviços de TI. Conforme avaliado por especialistas, o framework mostrou-se em conformidade com seus propósitos, dentre os quais destacamos o foco prático em "como fazer" e a adaptabilidade. Todos os aspectos avaliados (clareza, foco na qualidade, utilidade, adaptabilidade, entrega de resultado, adequação e abrangência, flexibilidade) receberam opiniões extremamente positivas. Isso nos leva a compreender que a proposta atingiu o seu objetivo, desenvolver o framework, e foi bem aceita na comunidade de especialistas.

Uma limitação que podemos destacar é que os especialistas não tiveram a oportunidade de utilizar o framework na prática, o que poderia resultar em uma avaliação mais apurada. Espera-se que o uso do framework nos IFs proporcione impactos positivos na satisfação do usuário do serviço. Como trabalho futuro, propõe-se a análise de viabilidade do framework em organizações privadas, e o seu aperfeiçoamento através de automatização do processo de implantação e da agregação de métodos para medir a maturidade da Central de Serviços.

Observamos que devido às características das Universidades Federais, que têm uma estrutura parecida com a dos IFs, provavelmente o framework possa ser utilizado com sucesso nestas instituições. Essa suposição é reforçada pelas características de adaptabilidade e flexibilidade do framework, todavia precisam ser realizados experimentos para comprová-la.

\section{Referências}

DE ARAUJO, L. L.; MOCNY, E. C.; ROCHA, A. R.; GONÇALVES, T.; SANTOS, G. (2014) "Experiência de Implantação do MR-MPS-SV no Service Desk da ECO Sistemas", VIII Simpósio Brasileiro de Qualidade de Software, Blumenau, Brasil.

ABNT. NBR ISO 20000-1:2011. Tecnologia da Informação - Gestão de Serviços. Parte 1: Requisitos do sistema de gestão de serviços. Rio de Janeiro, 2011.

ABNT. NBR ISO 9001:2015. Sistemas de gestão da qualidade: Requisitos. Rio de Janeiro, 2015.

BRASIL. 2015. Ministério da Educação. Expansão da Educação Superior e Profissional Tecnológica. Disponível em URL: http://redefederal.mec.gov.br/expansao-da-redefederal Acesso em: 27 de julho de 2015.

BRIGANO,G.U.; BARROS,R.M.(2010) “A Implantação de um Service Desk: Um Estudo de Caso Aplicando Conceitos do ITIL e do PMBOK”, IV Workshop Anual do MPS, Campinas, Brasil. 
CMMI Product Team. Cmmi for services, version 1.3 (cmu/sei-2010-tr-034). Technical report, Software Engineering Institute, Carnegie Mellon University, 2010.

FEIGENBAUM, Armand Vallin. Total quality control: engineering and management, the technical and managerial field for improving product quality, including its reliability, and for reducing operating cost and losses. New York. McGraw-Hill. 1961.

FERNANDES, Aguinaldo Aragon; ABREU, Vladimir Ferraz de. Implantando a governança de TI: da estratégia à gestão dos processos e serviços. Rio de Janeiro: Brasport, 2014.

ISHIKAWA, Kaoru. Controle de Qualidade Total: à maneira japonesa. Rio de Janeiro: Campus, 1993.

JÄNTTI, Marko; CATER-STEEL, Aileen; SHRESTHA, Anup. Towards an improved IT service desk system and processes: a case study. International Journal on Advances in Systems and Measurements, v. 5, n. 3 \& 4, p. 203-215, 2012.

JURAN, Joseph M. A qualidade desde o projeto: Novos passos para o planejamento da

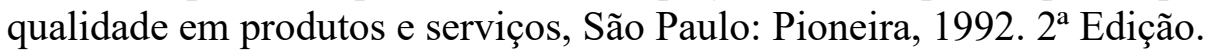

KELLER, Alexander; MIDBOE, Tyson. Implementing a service desk: A practitioner's perspective. In: Network Operations and Management Symposium (NOMS), 2010 IEEE. p. 685-696.

KRUCHTEN, Philippe. Introdução ao RUP: Rational Unified Process. Rio de Janeiro: Ciência Moderna, 2003.

MCFEELEY, B. IDEAL: A User's Guide for Software Process Improvement. Software Engineering Institute Handbook - sei.cmu.edu. Carnegie Mellon University Pittsburgh, Pennsylvania, 1996.

OGC. Office of Government Commerce. ITIL - The Official Introduction to the ITIL Service Lifecycle. London: TSO (The Stationary Office), 2007.

PMBOK, Guia. Um Guia do Conhecimento em Gerenciamento de Projetos. 5. ed. Project Management Institute, 2013.

RODRIGUES,G.R.; SOUZA,A. S., OLIVEIRA,L.O.(2013) "Implementando o Nível G do MR-MPS-SV com base no conceito de Central de Serviços", IV Workshop Anual do MPS, Campinas, Brasil.

RUDD, Collin. ITIL V3 Planning to Implement Service Management. TSO (The Stationary Office), 2010.

SOFTEX. Associação para Promoção da Excelência do Software Brasileiro. MPS.BR Guia Geral MPS de Serviços: 2015. Disponível em: $<\mathrm{http}: / /$ www.softex.br/mpsbr/guias/>. Acesso em: 21/12/2016.

TANG, X.; TODO, Y. (2013). A Study of Service Desk Setup in Implementing IT Service Management in Enterprises. Technology and Investment, Vol.4 No.3. Disponível em: http://www.scirp.org/journal/PaperInformation.aspx?PaperID=35498 